\title{
OXYGEN TOXICITY
}

\author{
Maj ANUJ CHAWLA * , Col AK LAVANIA +
}

\begin{abstract}
Oxygen, the gas vital to sustain life, can also destroy it. It may become toxic at an elevated partial pressure, which may be the result of a rise in inspired oxygen concentration, an increase in environmental pressure or a combination of both. The toxicity tends to express itself most recognizably in one of several forms including central nervous system manifestations, pulmonary signs and symptoms and ocular effects, especially in premature infants. Other forms of toxicity should be expected in severe exposure but are difficult to detect and hence are not often considered important limitations for the use of oxygen. The onset and degree of the toxicity vary with the concentration of the gas used and the duration of exposure. Marked variations in individual susceptibility are found. Prevention of its occurrence and early detection of toxicity are called for, the treatment being symptomatic.
\end{abstract}

MJAFI 2001; $57: 131-133$

KEY WORDS : Free radicals; Hyperbaric Oxygen Therapy (HBOT); Oxygen toxicity.

\section{Introduction}

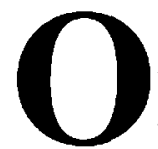

xygen has been present in atmosphere for 5 billion years. It is believed that its concentration in atmosphere was infinitesimal until 2.5 billion years ago when the first photosynthetic organisms made their appearance. The gradual increment in oxygen concentration in atmosphere allowed further evolution of animal species depending on aerobic metabolism. Those forms survived that had better protection against increasing oxidative stress of the changing atmosphere [1]. Priestley, who discovered oxygen, was himself amongst the first to suggest that there may be adverse affects of this 'pure air', when, in 1775, he observed a candle burn out faster in oxygen than in air and wondered if 'the animal powers be too soon exhausted in this pure kind of air' [2].

The first important contribution in field of oxygen toxicity was by Paul Bert who, in 1878, demonstrated convulsions in larks exposed to 15-20 ATA (atmosphere absolute) air [3]. The CNS toxic effects of oxygen are hence called 'Bert effect'. In 1899, J Lorain Smith, while trying to reproduce 'Bert effect', noticed fatal pneumonia in rats after 4 days of exposure to $73 \%$ oxygen at 1 ATA. This marked the discovery of pulmonary toxicity of oxygen, the 'Smith Effect' $[2,3]$.

The partial pressure of oxygen, in inspired air, at sea level is about $160 \mathrm{~mm} \mathrm{Hg}$. This value can be increased by either breathing $100 \%$ oxygen, thus theoretically increasing the $\mathrm{PO}_{2}$ of inspired air to $760 \mathrm{~mm}$ $\mathrm{Hg}$, or by increasing the pressure of the breathing mixture by which means there is, theoretically, no upper limit [4]. The re-emergence of hyperbaric oxygen therapy (HBOT), coupled with more aggressive oxygen therapy for pulmonary insufficiency, has caused large number of patients to be exposed to high oxygen pressures. Broadly two sets of clinical settings exist; one where the patient is exposed to very high concentrations of oxygen for brief periods of time (eg. HBOT) and the other where lower concentrations of the gas are used but for longer duration. These two lead to what has often been called 'acute' and 'chronic' oxygen toxicity respectively. The acute effects are predominantly CNS manifestations, while pulmonary features dominate chronic toxicity $[2,4,5]$.

\section{Central Nervous System Toxicity}

CNS toxicity, as described originally by Bert, occurred at oxygen pressures of $>3$ ATA. However, lower pressures may result in similar effects if exposure is prolonged, as shown in Fig 1. Early symptoms and signs are quite variable but twitching of perioral and small muscles of the hand is a fairly constant feature. Facial pallor and 'cogwheel' breathing are also often noticed, thought to be the result of intense peripheral vasoconstriction due to hyperoxia and diaphragmatic twitching respectively $[5,6]$. If exposure is continued, vertigo and nausea, followed by altered behaviour, clumsiness, and finally convulsions result. The convulsions begin with a loss of conciousness and develop in three phases; a tonic phase with a generalized hypertonus lasting for about $1 \mathrm{~min}$, a clonic phase with convulsion for about 2-3 mins and a post critical phase of about 10 mins. The patient has no memory of the crisis. A neurogenic pulmonary edema concomitant with the convulsions has also been reported [1]. CNS toxicity is hastened by factors such as raised

*Postgraduate trainee, 'Professor and Head, Department of Physiology, Armed Forces Medical College, Pune - 411040. 


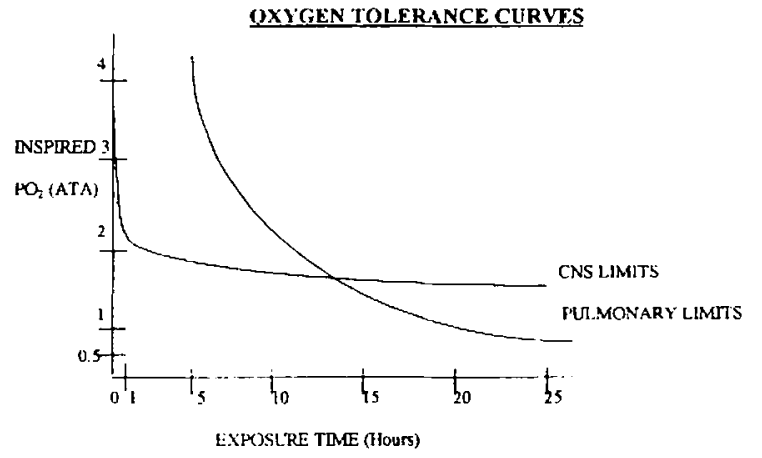

Fig. 1: Predicted human pulmonary and central nervous system tolerance to high pressure oxygen

$\mathrm{PCO}_{2}$, stress, fatigue and cold [2].

\section{Pulmonary Toxicity}

Pulmonary toxic effect of oxygen can arise after prolonged exposure to oxygen $>0.5$ ATA. Symptoms appear after a latent period whose duration decreases with increase in $\mathrm{PO}_{2}$. In normal humans the first signs of toxicity appear after about 10 hours of oxygen at 1ATA. Clinical features can be divided into three phases (a) Tracheobronchitis (b) ARDS (c) Pulmonary interstitial fibrosis. Absorption atelectasis due to washout of $\mathrm{N}_{2}$ can lead to collapse of parts of the lung in the event of air trapping.

$100 \%$ oxygen can be tolerated at sea level for about 24-48 hours without any serious tissue damage. Longer exposures produce definite tissue injury. Oxygen at 2 ATA produces characteristic pulmonary signs and symptoms beginning with mild carinal irritation on deep inspiration 3-6 hours into the exposure, intense carinal irritation an uncontrolled cough after about 10 hours and finally chest pain and dyspnoea. Symptoms subside 4 hours after cessation of exposure in majority of patients [7].

\section{Toxic Effects On Other Tissues}

Pulmonary and CNS effects, being most dramatic, are often the only ones to receive attention. Oxygen toxicity does affect other tissues also. Abnormal RBC morphology with or without a reduction in circulating mass of RBCs has been documented. There have been reports of occasional haemolytic episodes following HBOT [2]. Effects on eye include reversible constriction of the peripheral field of vision, a progressive myopia, which is also reversible, and cataract, which is a delayed manifestation. Ocular effects may be more when the entire eye is itself exposed to high ambient oxygen concentration and pressure, as in an oxygen tent, rather than when hyperoxia occurs via arterial circulation, (eg. following oxygen administra- tion via a facemask) [5]. Serous otitis media is seen in some aviators exposed to high concentrations of the gas. Dysbaric osteonecrosis in astronauts may also be partially contributed to by higher than normal levels of oxygen during space flights [2]. Neonates and premature infants exposed to high concentrations of oxygen are known to develop retinopathy, chronic lung disease and intraventricular haemorrhages. Premature infants of less than 30 weeks of gestation or $1500 \mathrm{~g}$ birth weight appear to be at a greater risk [8]. The critical oxygen concentration beyond which these conditions develop is $60 \%$.

\section{Pathophysiology}

It was as early as 1954 that Gerschman et al suggested oxygen derived free radicals as being the probable etiological factor in the development of these toxic effects [1]. Free radicals are defined as ions, atoms or molecules having an unpaired electron in an outer orbital [9]. These radicals are highly reactive owing to tendency of the radical to either add an electron to the unpaired one or to donate the extra electron. Both these processes result in generation of another free radical in the bargain. Free radical generation is a continuous process in the body as a result of oxireductive processes in the mitochondria where four electrons are added to each oxygen molecule and the product is two molecules of water [10]. This is the end result of a four-stage reaction, with an electron being added in each stage, producing superoxide $\left(\mathrm{O}_{2}\right)$, hydrogen peroxides $\left(\mathrm{H}_{2} \mathrm{O}_{2}\right)$, hydroxyl $(. \mathrm{OH})$ and $\mathrm{fi}^{-}$ nally water [1]. Free radicals are also produced by the action of enzymes such as xanthine/urate oxidase at extramitochondrial sites, from autooxidative reactions and by phagocytes during bacterial killing. These free radicals are known to cause lipid peroxidations, especially in the cell membranes, inhibit nucleic acids and protein synthesis and inactivate cellular enzymes. The body has a set of defense against these free radicals in the form of various antioxidant enzymes like glutathione peroxidase, catalase and superoxide dismutase. In hyperoxic conditions the explosive production of free radicals swamp enzyme systems and as a result free radicals escape inactivation.

This, however is not the only way by which oxygen causes toxicity. It can also exert non-radical mediated injury by altering cellular metabolism or by hyperoxia induced enzyme inhibition. Glutamic acid decarboxylase is one such enzyme inhibited in the CNS and reduced levels of Gama Amino Butyric Acid (GABA) are seen concomitantly with occurrence of seizures $[1,2]$. Post mortem studies in animals show selective 
grey matter and neuronal necrosis, with single short exposures producing ultrastructural changes in the anterior horn grey matter and severe prolonged exposures causing haemorrhagic necrosis in the brain at scattered or selective sites. The lungs reveal marked exudation with congestion and oedema, sometimes associated with intra-alveolar haemorrhages, necrosis of the alveolar cells and epithelial desquamation. The initial damage is at the level of capillary endothelium, followed by oedematous thickening of the alveolar septum, destruction of the type I pneumocytes, formation of hyaline membranes, intra-alveolar haemorrhages and areas of atelectasis $[1,11]$.

\section{Prevention and Monitoring}

The management of the condition being purely symptomatic, attention should be focused on its prevention. The abrupt stoppage of oxygen at the onset of toxicity may at times aggravate the symptoms, the 'oxygen off effect', and must be kept in mind [5]. An indicator used to monitor the pulmonary toxicity is the reduction in the vital capacity of the patient, which is known to occur. A $10 \%$ reduction is the maximum acceptable [5,7]. Dynamic lung compliance and the diffusing capacity for carbon monoxide $\left(D_{1} C O\right)$ are also seen to reduce.

Unit of Pulmonary Toxicity Dosage (UPTD) is a theoretical concept used to predict the extent of pulmonary damage with complicated and prolonged therapy. One minute of $100 \%$ oxygen at 1 atmosphere is taken to produce 1 UPTD. A UPTD of 1425 will produce a $10 \%$ reduction in the vital capacity [2].

Monitoring of CNS toxicity is based on calculations made from graphs that plot the CNS tolerance curves for humans (Fig-1). In lieu of a better index, the occurrence of a $10 \%$ incidence of neurological symptoms during exposure to 2.8 to 4 ATA has been taken to describe CNS tolerance in humans. EEG is of no value in the monitoring of patients [5].

The onset and rate of progression of this condition can be influenced by a variety of conditions, procedures and drugs. Induction of antioxidant enzymes, such as superoxide dismutase, by exposure to non-lethal levels of hyperoxia/hypoxia alone or in combination has been tried successfully in animals and is in the process of being evaluated in man. It is thought that this may lead to the deveiopment of tolerance to subsequent hyperoxic exposure [11].

Exogenous antioxidants, especially vitamin $\mathrm{E}$ and $\mathrm{C}$ have been found to reduce the incidence of retrolental fibroplasia in premature infants on hyperoxic therapy [5]. Adrenalectomy, hypophysectomy and the hypothyroid state are associated with reduced severity of the toxcity as is the use of alpha-adrenergic blockers [5,11]. Dietary deficiency of trace elements such as selenium, zinc and magnesium seem to worsen the toxicity, these being essential co-factors for the endogenous antioxidant enzymes [5,11]. Dietary supplementation has not been reported to confer any additional protection.

Oxygen is commonly used as part of the therapy of many disorders. This gas can have toxic effects if used injudiciously. These toxic manifestations usually involve the CNS, lungs and the eyes. The management of the condition is purely symptomatic and the emphasis should lie on its prevention. Individual susceptibility being very variable, it is imperative for all clinicians to be aware of and equipped to manage the crisis should it arise.

\section{References}

1. De Martino G, Luchetti, De Rosa RC. Toxic effects of oxygen. In:Michael M, Marroni A, Longoni C, Editors. Handbook of Hyperbaric Medicine. New York; Springer. 1996;59-68.

2. Edmonds C,Lowry C, Pennefather J. Oxygen Toxicity. In : Edmonds C, Lowry $\mathrm{C}$ and Pennefather J, Editors. Diving and Subaquatic Medicine. Oxford; Butterworth-Heinemann. 1992;241-56.

3. Donald $\mathrm{KW}$. Oxygen poisioning in man (1,II). BMJ 1947;667-72,712-7.

4. Miles S. Oxygen. In:Miles S, Editor.Underwater Medicine. London;Staples 1969.128-51.

5. Clark JM.Oxygen toxicity. In:Bennitt PB, Elliot DH, Editors. The Physiology and Medicine of Diving.London;BailliereTindall. 1982;200-38.

6. Lambertson CJ. Effects of excessive pressures of oxygen, nitrogen, helium, carbon dioxide, and carbon monoxide. In Mountcastle VB. Editor. Medical Physiology. Missouri; CV Mosby Co. 1980;1901-46.

7. Clark JM, Lambertson CJ. Pulmonary oxygen tolerance and the rate of development of pulmonary oxygen toxicity in man at two atmospheres inspired oxygen tension. In:Lambertson CJ, Editor. Underwater Physiology; Proceedings of the third symposium.Baltimore Williams and Wilkins. 1967:439-51.

8. Kelly FJ. Free radical disorders of preterm infants.British Medical Bulletin 1993;49(3):481-93.

9. Cheeseman KH, Slater TF. An introduction to free radical biochemistry. British Medical Bulletin 1993:49(3):481-93.

10. Fridovich I. The biology of free radicals. Science 1978:201:875-80.

11. Deneke SM, Fanburg BL. Normobaric oxygen toxicity of the lung. N Engl J Med 1980;303(2):76-86. 